\title{
Linguistic Validation of the Turkish Version of the M.D. Anderson Symptom Inventory - Head and Neck Cancer Module
}

\author{
G. Brandon Gunn ${ }^{1}$, Banu Atalar ${ }^{2}$, Tito R. Mendoza ${ }^{3}$, Charles S. Cleeland ${ }^{3}$, Uğur Selek ${ }^{1,4}$, Enis Özyar ${ }^{2}$, \\ David I. Rosenthal ${ }^{1}$
}
${ }^{1}$ Department of Radiation Oncology, The University of Texas MD Anderson Cancer Center, Texas, USA ${ }^{2}$ Department of Radiation Oncology, Acıbadem University School of Medicine, İstanbul, Turkey ${ }^{3}$ Department of Symptom Research, The University of Texas MD Anderson Cancer Center, Texas, USA ${ }^{4}$ Department of Radiation Oncology, Koç University School of Medicine, İstanbul, Turkey

Background: The use of patient symptom reports with frequent symptom assessment may be preferred over the more commonly used health-related quality of life questionnaires.

Aims: We sought to linguistically validate the Turkish version of the M.D. Anderson Symptom InventoryHead and Neck module (MDASI-HN) patient reported outcome questionnaire.

Study Design: Validation study.

Methods: Following standard forward and backward translation of the original and previously validated English MDASI-HN into a Turkish version (T-MDASI$\mathrm{HN}$ ), it was administered to patients with head and neck cancer able to read and understand Turkish. Patients were then cognitively debriefed to evaluate their understanding and comprehension of the T-MDASI-HN. Individual and group responses are presented using descriptive statistics.
Results: Twenty-six participants with head and neck cancer completed the T-MDASIHN and accompanying cognitive debriefing. Overall, 97 percent of the individual TMDASI-HN items were completed. Average recorded time to complete the 28 item TMDASI-HN questionnaire was 5.4 minutes (range 2-10). Average overall ease of completion, understandability, and acceptability were favorably rated at $1.0,1.1$, and 0.2 , respectively, on scales from 0 to 10 . Only 5 of the 26 of participants reported trouble completing any single questionnaire items, namely the "difficulty remembering" item for 3 individuals.

Conclusion: The T-MDASI-HN is linguistically valid with ease of completion, relevance, comprehensibility, and applicability and it can be a useful clinical and research tool.

Keywords: Head and neck cancer, patient-reported questionnaire, patient symptoms, Turkish MDASI-HN
Patients with cancer often experience substantial tumorand/or treatment-related symptoms, which can impact their overall wellbeing and function (1). Achieving optimal symptom control can be challenging and requires ongoing symptom assessment across the entirety of the disease and treatment course. Considering the patient experience via patient reported outcome instruments, rather than relying on traditional physician ratings of toxicity alone, is now increasingly emphasized in both patient care and clinical research (2). Furthermore, the use of patient symptom reports with frequent symptom assess- ment may be preferred over the more commonly used healthrelated quality of life questionnaires, as patient symptoms are felt to more closely reflect the dynamic changes encountered during cancer treatments and/or during the disease process itself, thus giving providers pertinent and actionable clinical information (3).

The M.D. Anderson Symptom Inventory (MDASI) is a brief, reliable, and validated patient-reported multi-symptom assessment tool that quantifies common cancer- and therapyrelated symptoms. It contains 13 "core items" representing 
important symptoms common across all cancer types and 6 items on how these symptoms interfere with major activities of daily functioning (4). Multiple non-English versions of the MDASI have been previously psychometrically validated in terms of content, construct, reliability, and known group validity (5-10).

The MDASI was initially constructed so that subsequent specific cancer type and treatment site modules could be developed. For a given tumor's anatomic location and depending on local tumor extent, patients with head and neck cancer can experience a number of unique, bothersome and potentially threatening symptoms. Furthermore, patients with head and neck cancer are commonly treated with a combined modality approach (combinations of chemotherapy, surgery, and/ or radiation), which are known to be associated with substantial acute and long term toxicity and functional impact. Therefore, the MDASI-Head and Neck module (MDASI-HN) was developed and validated as a disease-site specific instrument, inclusive of the initial 13 core or general cancer items and 6 interference items, with an additional 9 "head and neck specific items" that represent both the tumor- and treatmentrelated toxicities symptoms that were determined to be most important to head and neck cancer patients, some of which were unrepresented by other instruments (11), including key mucositis-associated symptoms, which correlated strongly with observer rated mucositis severity (12). Using the MDASI-HN, we have previously shown that the head and neck cancer patient symptom profile both before radiation-based treatment has begun (13), during and toward treatment end can be uniquely profiled (14) and the severity of the symptom experience predicted (15). The MDASI and MDASI-HN patient reported outcome tools are currently being used in the Head and Neck Center outpatient clinics at MD Anderson Cancer Center as part of our routine new patient evaluation process, as patient management tools during and after therapy completion, and as part of ongoing practice quality improvement efforts.

In order to allow integration of the MDASI-HN as a clinical assessment tool in primary Turkish-speaking regions and for Turkish-speaking patients, and to ensure the inclusion of these patients in symptom prevention and intervention clinical studies that use the MDASI-HN as an endpoint measure, our goal is to linguistically validate the Turkish version of the MDASIHN (T-MDASI-HN).

\section{MATERIALS AND METHODS}

The MDASI-HN was translated into Turkish language using standard forward and backward translation methods, procedures that we have been following as necessary first steps prior to use or psychometric validation of any non-English language version of the MDASI or its modules $(5-10,16)$, as recommended by an international task force (17).

The protocol was approved by the independent ethics committee and written informed consent was obtained for each patient. Following approval from the local institution ethics board, consecutive adult patients with malignancy of the head and neck region, able to read and understand Turkish, were recruited in our department. The T-MDASI-HN was selfadministered by the participating patients. All T-MDASI-HN symptom items are rated on 0 to 10 numeric scales from "not present" to "as bad as you can imagine", and the T-MDASI$\mathrm{HN}$ interference items are rated on 0 to 10 numeric scales from "did not interfere" to "interfered completely." Time taken by each participant to complete the T-MDASI-HN was recorded by clinical staff. Since the purpose of this study was purely linguistic validation, patient demographic, tumor, and treatment details were not recorded.

To ensure the ease of completion, relevance, and comprehensibleness of this translated version, and in keeping with recent recommendations, participants also completed a cognitive debriefing of the T-MDASI-HN $(17,18)$. The cognitive debriefing was completed with the assistance of clinic staff, who were both Turkish and English speaking. Subjects were asked to rate overall ease of completion, understandability, and ease of severity rating recall of the T-MDASI-HN, asked whether they were comfortable answering each specific item, whether any item was unclear, and whether they had any suggestions on how to make any question clearer or better. Subjects were also asked whether any item was redundant, whether any item should be deleted, or whether any specific item should be added. The overall ease of completion and understandability/comprehensibility of the T-MDASI-HN and ease of severity rating recall were rated on scales from 0 to 10 with " 0 " being "very easy" and " 10 " being "very hard", and the overall acceptability and comfort level of the patient with the questionnaire was rated on a scale from 0 to 10 with " 0 " being "very comfortable" and " 10 " being "very uncomfortable". Here, we present the T-MDASI-HN item severity and response rate along with the cognitive debriefing results using descriptive statistics. All statistical analyses were done with JMP version $9\left({ }^{\circ} 2013,1998\right.$ 2012, SAS Institute Inc; Cary, NC, USA).

\section{RESULTS}

From 03/12 through 03/13, 26 head and neck cancer patients previously treated at our department participated in and completed the T-MDASI-HN along with the accompanying cognitive debriefing. Overall, 707 of the possible 728 (97\%) 
TABLE 1. Turkish M.D. Anderson Symptom Inventory-Head and Neck module severity ratings and missing values per questionnaire item

\begin{tabular}{|c|c|c|c|}
\hline & $\begin{array}{c}\text { Mean Rating* } \\
\text { (Standard } \\
\text { Deviation) }\end{array}$ & Range & $\begin{array}{c}\text { Missing Values } \\
\text { (not completed } \\
\text { by patient) }\end{array}$ \\
\hline \multicolumn{4}{|l|}{ CORE ITEMS } \\
\hline Pain & $2.2(2.6)$ & $0-8$ & $1 / 26$ \\
\hline Fatigue & $2.8(2.7)$ & $0-9$ & $0 / 26$ \\
\hline Nausea & $1.5(3.2)$ & $0-10$ & $1 / 26$ \\
\hline Sleep disturbance & $2.2(2.9)$ & $0-9$ & $1 / 26$ \\
\hline Distress & $2.3(2.7)$ & $0-10$ & $0 / 26$ \\
\hline Shortness of breath & $1.2(2.2)$ & $0-9$ & $0 / 26$ \\
\hline Difficulty remembering & $1.5(2.2)$ & $0-8$ & $0 / 26$ \\
\hline Lack of appetite & $2.1(2.4)$ & $0-9$ & $0 / 26$ \\
\hline Drowsiness & $2.3(2.5$ & $0-8$ & $0 / 26$ \\
\hline Dry mouth & $4.3(3.6)$ & $0-10$ & $0 / 26$ \\
\hline Sadness & $1.8(2.4)$ & $0-8$ & $0 / 26$ \\
\hline Vomiting & $0.8(2.3)$ & $0-9$ & $1 / 26$ \\
\hline Numbness/tingling & $1.0(2.1)$ & $0-8$ & $0 / 26$ \\
\hline \multicolumn{4}{|l|}{ HEAD AND NECK ITEMS } \\
\hline Problem with mucus in mouth/throat & $3.7(3.0)$ & $0-9$ & $1 / 26$ \\
\hline Difficulty swallowing/chewing & $2.1(2.7)$ & $0-10$ & $0 / 26$ \\
\hline Choking & $2.1(2.7)$ & $0-9$ & $1 / 26$ \\
\hline Difficulty with voice/speech & $2.4(2.8)$ & $0-9$ & $0 / 26$ \\
\hline Skin pain/burning/rash & $1.0(1.8)$ & $0-6$ & $1 / 26$ \\
\hline Constipation & $1.7(2.3)$ & $0-6$ & $9 / 26$ \\
\hline Problem tasting food & $2.6(3.2)$ & $0-10$ & $0 / 26$ \\
\hline Mouth/throat sores & $2.4(3.3)$ & $0-10$ & $1 / 26$ \\
\hline Problem with teeth/gums & $2.0(3.0)$ & $0-10$ & $0 / 26$ \\
\hline \multicolumn{4}{|l|}{ SYMPTOM INTERFERENCE } \\
\hline General activity & $2.1(2.3)$ & $0-9$ & $1 / 26$ \\
\hline Mood & $2.0(2.5)$ & $0-9$ & $0 / 26$ \\
\hline Work & $1.7(2.7)$ & $0-9$ & $0 / 26$ \\
\hline Relations with other people & $1.3(2.2)$ & $0-8$ & $1 / 26$ \\
\hline Walking ability & $1.5(2.3)$ & $0-7$ & $1 / 26$ \\
\hline Enjoyment of life & $1.2(2.4)$ & $0-10$ & $1 / 26$ \\
\hline
\end{tabular}

*Rated on a $0-10$ scale with higher ratings being more severe.

individual T-MDASI-HN items were completed by the patients. The average time to complete the T-MDASI-HN was 5.4 minutes (standard deviation (SD): 1.9; range 2-10). The TMDASI-HN symptom and interference severity ratings along with tabulation of missing values per questionnaire item are shown in Table 1.
TABLE 2. Cognitive debriefing results for the Turkish M.D. Anderson Symptom Inventory-Head and Neck module and missing values per debriefing item

\begin{tabular}{lccc}
\hline & $\begin{array}{c}\text { Mean Rating* } \\
\text { (Standard Deviation) }\end{array}$ & $\begin{array}{c}\text { Missing Values } \\
\text { (not completed) }\end{array}$ \\
\hline COGNTIVE DEBRIEFING ITEMS & $1.0(1.5)$ & $0-5$ & $0 / 26$ \\
Ease of completion & $1.1(1.2)$ & $0-5$ & $2 / 26$ \\
Understandability & $0.2(0.5)$ & $0-2$ & $0 / 26$ \\
Acceptability & $0.6(1.0)$ & $0-4$ & $1 / 26$ \\
Ease of 24 hour severity level recall &
\end{tabular}

*Rated on a 0-10 scale with higher ratings being more severe.

The key components of the cognitive debriefing results are presented in Table 2 . The majority of participants $(21 / 26)$ thought that all T-MDASI-HN items, question, phrases, and words were easy to understand. Of the remaining 5, 3 subjects reported difficulty with the "difficulty remembering" item. Other individual items rated with at least some difficulty in understanding were "choking/coughing" (1 participant), "sad" (1 participant), and "pain" (1 participant). No participant reported any difficulty understanding any of the interference items. No subject thought that any specific item should be deleted, and 8 participants provided suggestions to add one or more additional symptoms items to the questionnaire, including ear and/or hearing-related items (5 participants), "physiologic support" (1 participant), hair loss-related items (1 participant), smell-related items (1 participant), additional mood-related items (2 participants), and bone pain-related items (1 participant).

\section{DISCUSSION}

Here, we report the linguistic validation results of the TMDASI-HN. The favorable cognitive debriefing findings suggest overall ease of completion, relevance, acceptability and comprehensibility of this translated patient-reported outcome questionnaire in this Turkish head and neck cancer patient population. A few observations require further discussion. Problems with "constipation" was left blank by $9 / 26$ participants. We believe this is simply due to a paper questionnaire formatting issue, which has been remedied in the final and currently used questionnaire version. Further support for this observation being due to a formatting issue is the fact that no participant reported any difficulty understanding the "constipation" item on cognitive debriefing. Regarding cognitive debriefing results, 3 of 26 subjects reported difficulty understanding the individual item related to "difficulty remembering". Since this item is part of the 13 core items from the MDASI, which has been previously shown in a larger study of Turkish speaking 
subjects to demonstrate single item reliability (19), we continue to include this particular item and phrasing in our current T-MDASI-HN version. Notably, only one participant reported any difficulty understanding any single head and neck specific symptom item. Furthermore, we plan to explore the addition of ear/hearing-related items in future development and validation studies.

In conclusion, the T-MDASI-HN is a linguistically valid disease site-specific module of the MDASI with ease of completion, relevance, and comprehensibility and applicability. It can be useful in patient-reported outcomes research and as a simple clinical tool toward improved patient symptom assessment and management for Turkish speaking patients. The T-MDASI-HN and MDASI-HN patient reported outcome instruments are currently used in routine clinical practice at our institutions as patient management tools before, during and after therapy completion.

Ethics Committee Approval: Ethics committee approval was received for this study from the Acibadem University Ethics Committee (Date: 18 December 2012, Number: 2012-427).

Informed Consent:. Written informed consent was obtained from patients who participated in this study.

Peer-review: Externally peer-reviewed.

Author contributions: Concept - G.B.G., C.S.C., T.R.M., D.I.R.; Design - T.R.M., C.S.C.; Supervision - U.S., G.B.G., D.I.R.; Resource - E.O., B.A.; Materials - E.O., B.A.; Data Collection and/or Processing - E.O., B.A.; Analysis and/or Interpretation - E.O., B.A.; Literature Search - G.B.G., C.S.C.; Writing - G.B.G., E.O., D.I.R., U.S.; Critical Reviews - G.B.G., B.A., T.R.M., C.S.C., U.S., E.O., D.I.R.

Acknowledgements: The authors would like to express their sincere gratitude to the medical staff of the radiation oncology departments for their kind assistance in acquisition of data.

Conflict of Interest: No conflict of interest was declared by the authors.

Financial Disclosure: The authors declared that this study has received no financial support.

\section{REFERENCES}

1. Rosenthal DI, Mendoza TR, Fuller CD, Hutcheson KA, Wang XS, Hanna EY, et al. Patterns of symptom burden during radiotherapy or concurrent chemoradiotherapy for head and neck cancer: a prospective analysis using the University of Texas MD Anderson Cancer Center Symptom Inventory-Head and Neck Module. Cancer 2014;120:1975-84. [CrossRef]
2. Basch E, Abernethy AP, Mullins CD, Reeve BB, Smith ML, Coons SJ, et al. Recommendations for incorporating patient-reported outcomes into clinical comparative effectiveness research in adult oncology. J Clin Oncol 2012;30:4249-55. [CrossRef]

3. Cleeland CS, Sloan JA. Assessing the Symptoms of Cancer Using Patient-Reported Outcomes (ASCPRO): searching for standards. $J$ Pain Symptom Manage 2010;39:1077-85. [CrossRef]

4. Cleeland CS, Mendoza TR, Wang XS, Chou C, Harle MT, Morrisey $\mathrm{M}$, et al. Assessing symptom distress in cancer patients: the M.D. Anderson Symptom Inventory. Cancer 2000;89:1634-46. [CrossRef]

5. Ivanova MO, Ionova TI, Kalyadina SA, Uspenskaya OS, Kishtovich AV, Guo H, et al. Cancer-related symptom assessment in Russia: validation and utility of the Russian M. D. Anderson Symptom Inventory. J Pain Symptom Manage 2005;30:443-53. [CrossRef]

6. Lin C-C, Chang A-P, Cleeland CS, Mendoza TR, Wang XS. Taiwanese version of the M. D. Anderson symptom inventory: symptom assessment in cancer patients. J Pain Symptom Manage 2007;33:180-8. [CrossRef]

7. Mystakidou K, Cleeland C, Tsilika E, Katsouda E, Primikiri A, Parpa E, et al. Greek M.D. Anderson Symptom Inventory: validation and utility in cancer patients. Oncology 2004;67:203-10. [CrossRef]

8. Okuyama T, Wang XS, Akechi T, Mendoza TR, Hosaka T, Cleeland CS, et al. Japanese version of the MD Anderson Symptom Inventory: a validation study. J Pain Symptom Manage 2003;26:1093-104. [CrossRef]

9. Wang XS, Wang Y, Guo H, Mendoza Tr, Hao XS, Cleeland CS. Chinese version of the M. D. Anderson Symptom Inventory: validation and application of symptom measurement in cancer patients. Cancer 2004;101:1890-901. [CrossRef]

10. Yun YH, Mendoza TR, Kang IO, You CH, Roh JW, Lee CG, et al. Validation study of the Korean version of the M. D. Anderson Symptom Inventory. J Pain Symptom Manage 2006;31:345-52. [CrossRef]

11. Rosenthal DI, Mendoza TR, Chambers MS, Asper JA, Gning I, Kies MS, et al. Measuring head and neck cancer symptom burden: the development and validation of the M. D. Anderson symptom inventory, head and neck module. Head Neck 2007;29:923-31. [CrossRef]

12. Rosenthal DI, Mendoza TR, Chambers MS, Burkett VS, Garden AS, Hessell AC, et al. The M. D. Anderson symptom inventoryhead and neck module, a patient-reported outcome instrument, accurately predicts the severity of radiation-induced mucositis. Int J Radiat Oncol Biol Phys 2008;72:1355-61. [CrossRef]

13. Gunn GB, Mendoza TR, Fuller CD, Gning I, Frank SJ, Beadle BM, et al. High symptom burden prior to radiation therapy for head and neck cancer: a patient-reported outcomes study. Head Neck 2013;35:1490-8.

14. Shi Q, Mendoza TR, Gunn GB, Wang XS, Rosenthal DI, Cleeland CS. Using group-based trajectory modeling to examine heterogeneity of symptom burden in patients with head and neck 
cancer undergoing aggressive non-surgical therapy. Qual Life Res 2013;22:2331-9. [CrossRef]

15. Sheu T, Fuller CD, Mendoza TR, Garden AS, Morrison WH, Beadle BM et al. Nomogram for Predicting Symptom Severity during Radiation Therapy for Head and Neck Cancer. Otolaryngol Head Neck Surg 2014;151:619-26. [CrossRef]

16. Gunn GB, Koukourakis MI, Mendoza TR, Cleeland CS, Rosenthal DI. Linguistic validation of the Greek M.D. Anderson Symptom Inventory - Head and Neck Module. Forum Clin Oncol 2012;3:29-31.

17. Wild D, Grove A, Martin M, Eremenco S, McElroy S, VerjeeLorenz A, et al. Principles of Good Practice for the Translation and Cultural Adaptation Process for Patient-Reported Outcomes
(PRO) Measures: report of the ISPOR Task Force for Translation and Cultural Adaptation. Value Health 2005;8:94-104. [CrossRef]

18. U.S. Department of Health and Human Services FDA Center for Drug Evaluation and Research; U.S. Department of Health and Human Services FDA Center for Biologics Evaluation and Research; U.S. Department of Health and Human Services FDA Center for Devices and Radiological Health. Guidance for industry: patient-reported outcome measures: use in medical product development to support labeling claims: draft guidance. Health Qual Life Outcomes 2006;4:79. [CrossRef]

19. Karabulut N, Erci B, Ozer N, Ozdemir S. Symptom clusters and experiences of patients with cancer. $J A d v$ Nurs 2010;66:1011-21.[CrossRef] 